\title{
Observations of magnetic CP stars
}

\author{
T. Ryabchikova ${ }^{1,2}$ \\ ${ }^{1}$ Institute of Astronomy, Russian Academy of Science, 48 Pyatnitskaya str., 119017 Moscow, \\ Russia \\ email:ryabchik@inasan.rssi.ru \\ ${ }^{2}$ Institute for Astronomy, Vienna University, Türkenschanzstraße 17, A-1180 Vienna, Austria
}

\begin{abstract}
Important results of magnetic CP star research in the last decade from photometric and spectroscopic observations are discussed. Hipparcos parallaxes confirm CP stars are Main Sequence stars. Photometric monitoring of the rapidly rotating stars provides evidences for the rotational braking on the Main Sequence. High signal-to-noise spectra with high resolution and time-resolution give strong support for chemical separation processes operating in stellar atmospheres (abundance stratification). There are also observational evidences for the departure of the temperature structure of cool CP star atmospheres from that for normal stars.
\end{abstract}

Keywords. Stars: photometry, stars: spectroscopy, stars: chemically peculiar.

\section{Introduction}

Due to the growing power of modern telescopes on Earth and in space astronomers now have an opportunity to study the phenomenon of magnetic (and nonmagnetic) chemically peculiar (CP) stars in greater detail. We penetrate deeper into the Ap star puzzle. The development of observational techniques and research methods permit us to solve old problems and contradictions, providing us at the same time with new ones. In the next sections I will try to review briefly the latest important results obtained from the observations of magnetic CP (CP2) stars.

\section{Evolutionary status of magnetic CP stars from observations}

The analysis of Hipparcos parallaxes for CP stars (Gomez et al. 1998) showed that these stars are uniformly distributed across the whole width of the Main Sequence (MS), and their distribution does not differ from normal stars. Based also on Hipparcos data, but exploiting a specifically chosen group of CP2 stars (stars with $M \leqslant 3 M_{\odot}$, with resolved Zeeman Splitting in non-polarized spectra, and with small rotational velocities), Hubrig et al. (2000a) concluded that a star posesses a substantial magnetic field and chemical peculiarites only after it has completed at least $30 \%$ of its MS lifetime. An obvious contradiction between the two studies was successfully overcome by the recent investigations of the CP2 stars in open clusters. Pöhnl et al. (2003) on the basis of $\Delta a$ photometry classified a few CP stars in young open cluster NGC $2516(\log t \approx 8.1)$, and Bagnulo et al. (2003) have measured a strong magnetic field $B_{\mathrm{s}}=14.5 \mathrm{kG}$ in one of these stars, HD 66318, which has completed less than $16 \%$ of MS lifetime. The survey of magnetic fields in stars in open clusters with the FORS1 multi-object spectrograph revealed another supermagnetic $\left(B_{\ell}=-9 \mathrm{kG}\right)$ CP star NGC 2244-334 in the Rosette Nebula cluster, which has completed less than $3 \%$ of its MS lifetime (Bagnulo et al. 2004). These direct observations show that a star reaches ZAMS with slow rotation and significant magnetic field in agreement with the scenario proposed by Stȩpień (2000) to explain the slow rotation of CP2 stars, where magnetic fields in Bp-Ap stars are already present in the pre-Main Sequence phases. 


\section{Period changes}

What is going on with a magnetic CP star on the MS ? Does it undego the further magnetic braking? According to scenario by Stępień (2000) only stars with large magnetic fields become slow rotators, and they loose their angular momentum before the ZAMS. However, there are stars with typical CP characteristics, slow rotation and very weak magnetic fields which are already finished their MS lifetime. One example is HD 204411 (Ryabchikova et al. 2005) in which magnetic field does not exceed by much its equipotential value. The star might originally be a slow rotator, or seen pole-on, or it lost somehow its angular momentum during the MS stage. Although statistical investigations of rotational velocities in A stars give evidence for the conservation of angular momentum throughout the whole MS lifetime (see, e.g., Hubrig et al. 2000b), at present we have two CP2 stars, CU Vir=HD 124424 and 56 Ari=HD 19832 with observed retardations of their rotation. Both are among the fastest rotators of the magnetic CP stars. CU Vir suddenly changed its rotation period $\mathrm{P}=0.52$ day by only $2 \mathrm{sec}$ in 1984 (Pyper et al. 1998). This is sufficient to cause a half period phase change ten years later. The second star, 56 Ari, $\mathrm{P}=0.72$ day, demonstrated a constant retardation by $2 \mathrm{sec}$ over 100 years (Adelman et al. 2001). With such an angular momentum loss, a star will be a typical slow rotating CP star by the end of its MS lifetime. Both studies were based on careful analysis of all photometric and spectroscopic observations over 40 years, and were successful due to photometric monitoring with the FCAPT (Four College Automatic Photometric Telescope). Is the observed loss of the angular momentum typical for other rapidly rotating CP2 stars? Photometric monitoring of short-period CP stars with automatic telescopes will provide very important information for the evolutionary studies of CP stars (see also Pyper \& Adelman 2005).

\section{Spectroscopy: abundance stratification}

Analyses of the high resolution spectroscopic observations of the magnetic CP stars in wide spectral regions clearly show the presence of abundance stratifications in their atmospheres. From the early 1970s a competition between radiative acceleration and gravitational settling in stabilized atmosphere has been considered as the primary mechanism responsible for the observed abundance anomalies in CP stars (Michaud 1970) but detailed abundance startification studies began with the pioneer work by Babel (1992). His theoretical diffusion calculations showed that abundance profile may be represented by a step function with the steep abundance gradient. All empirical abundance stratification studies are based on this simple model (Babel (1992) 53 Cam, Ryabchikova et al. (2002) $\gamma$ Equ, Wade et al. (2003) $\beta$ CrB, Ryabchikova et al. (2005) HD 133792, HD 188041, and HD 204411). I plotted Cr and Fe empirical stratifications in these five stars in Fig. 1. The abundance jump occurs in a rather narrow range of optical depths $-1.5 \leqslant \tau_{5000} \leqslant-0.5$, and the position of this jump moves upwards with temperature. Abundance limits are also greater for higher temperatures. There is only a small difference in abundance startifications for stars with similar temperatures and very different magnetic field strength (HD 188041 and HD 133972, see Ryabchikova et al. 2005). However, significant differences exist for stars with comparable magnetic field strengths, but with different temperatures ( $\gamma$ Equ and HD 188041). The process of element separation in stellar atmospheres is governed by the temperature rather than by the magnetic field.

Five stars, of course, is insufficient for statistics, but we may look at the whole picture using averaged abundances which are available for many more stars and which roughly represent element abundances near the middle of abundance jump. The distribution of averaged $\mathrm{Cr}$ and $\mathrm{Fe}$ abundances with effective temperatures for different group of A stars 


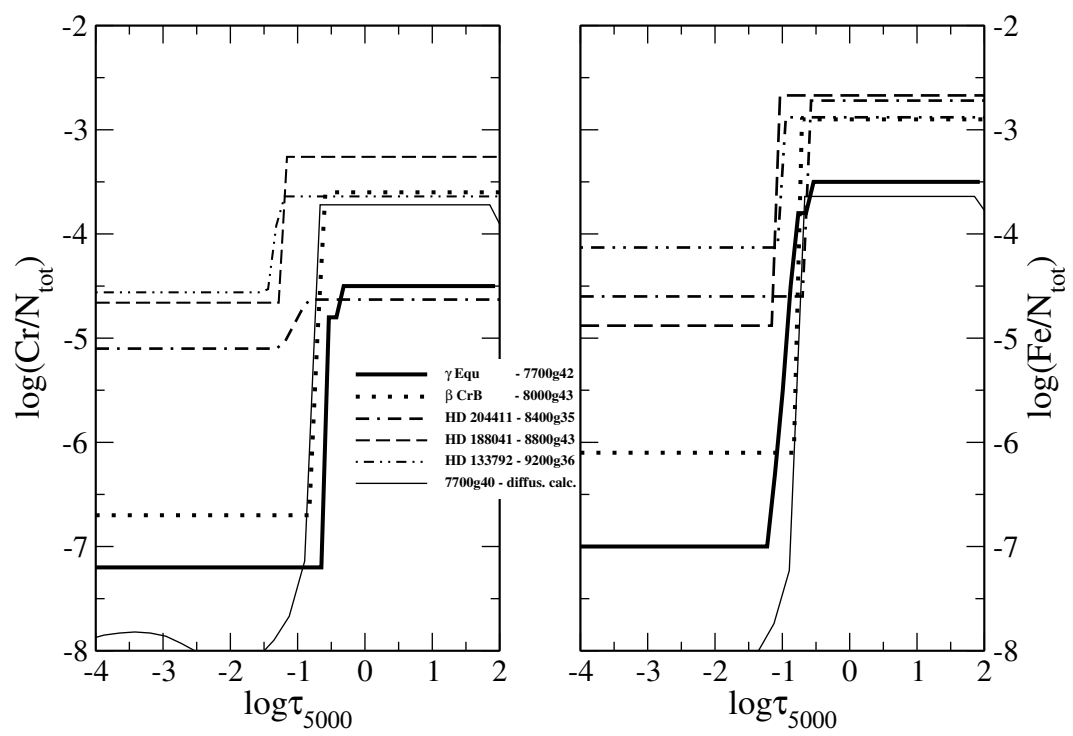

Figure 1. Element distribution in the atmospheres of Ap stars and comparison with self-consistent diffusion model predictions.
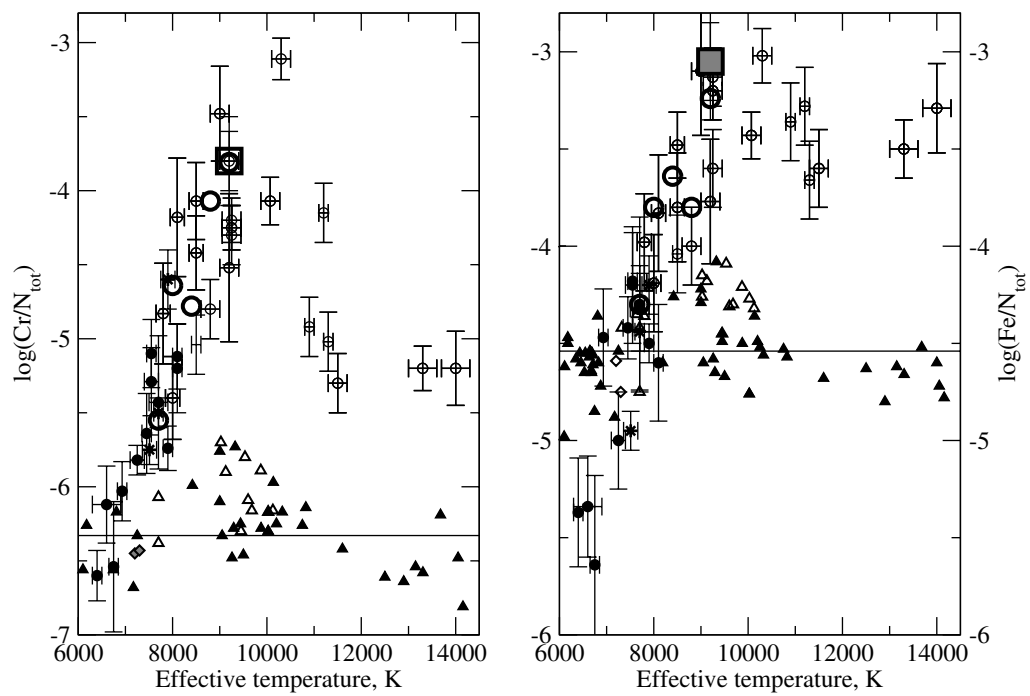

Figure 2. Cr (left panel) and Fe (right panel) average abundances versus effective temperature for 13 roAp stars (filled circles), 23 non-pulsating Ap stars (open circles) and 4 spectroscopic roAp candidates: HD 29578, HD 75445, HD 62140, and HD 115708 (asterisks). Solar abundances are indicated by a horizontal line. Five stars for which stratification analyses are available are shown by large open circles, and HD 66318 by a large open square.

are shown in Fig. 2. I add 3 additional stars to this plot, taken from Ryabchikova et al. (2004a - Fig. 5), HD 133792, HD 204411 and HD 66318. For the last star with a very large magnetic field $14.5 \mathrm{kG}$, abundance data are taken from Bagnulo et al. (2003). The five stars with abundance stratification analyses are shown by large open circles, and HD 66318 by a large open square. All six stars follow the overall distribution, where CP stars with very different magnetic fields are plotted. Fig. 2 seems to support the main role of the temperature in the process of element separation in CP star atmospheres. 
Stratification analyses of the CP stars with a wider range of effective temperatures and magnetic field strengths is required for a better understanding the separation processes.
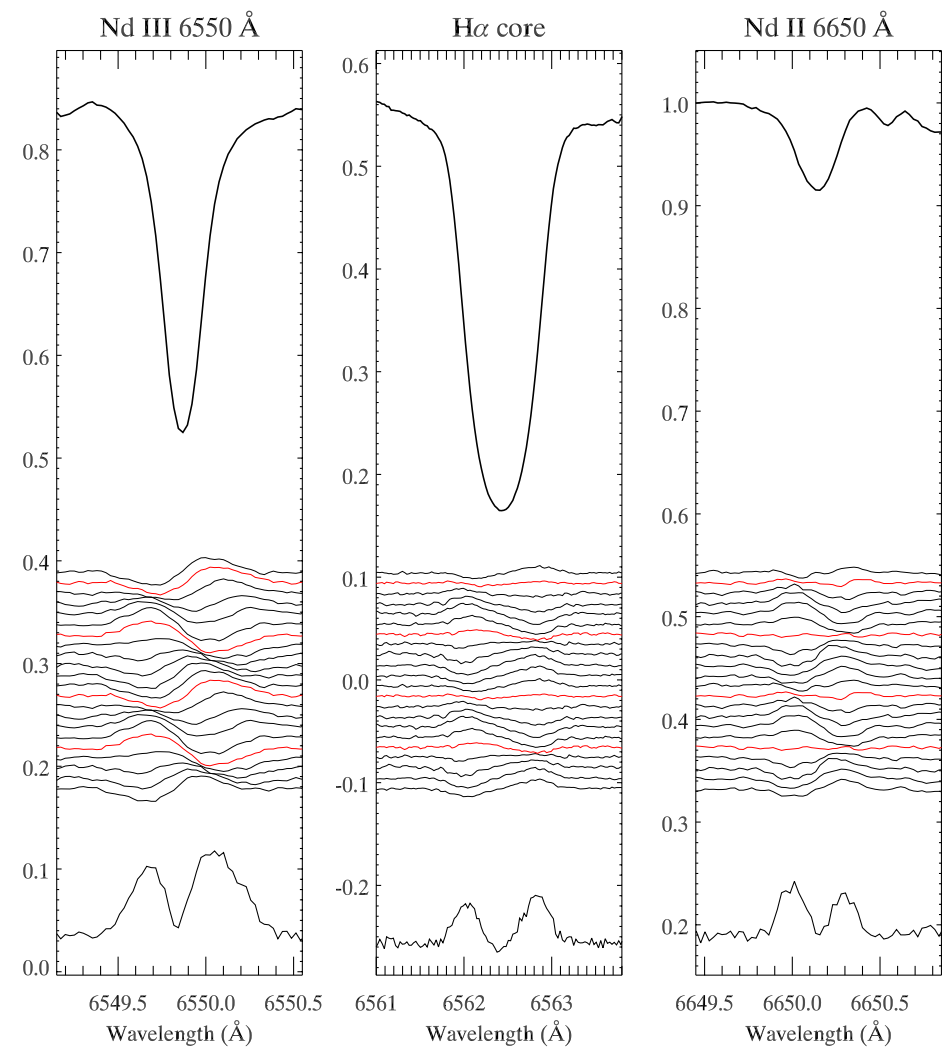

Figure 3. Pulsational line provile variations in spectrum of $\gamma$ Equ (CFHT observations, 2001). Different behaviour of the residual spectra are easily seen.

Empirical abundance stratifications derived in CP stars are supported by the results of the first self-consitent model calculations, which include radiative diffusion processes for 39 chemical elements (LeBlanc 2003, Ryabchikova et al. 2003, LeBlanc \& Monin 2005). We simultaneously get the abundance stratifications and the atmospheric model, calculated with stratified chemical composition. Model $\mathrm{Cr}$ and Fe distributions discussed by Leblanc \& Monin (2005) are plotted in Fig. 1 together with the empirical distributions. The model confirms the realibility of the empirical abundance distributions. Thus empirically derived stratifications are not an artefact, caused by poor knowledge of atmospheric structure in such complex objects as magnetic CP stars. Further we may extend the empirical stratification analysis to the elements not yet calculated. Rare-earth elements (REE) are the most important, because their lines provide extremely important information about the pulsations in the atmospheres of cool CP2 stars (Savanov et al. 1999, Kochukhov \& Ryabchikova 2001, Ryabchikova et al. 2002, Kurtz 2005). In spectra of rapidly oscillating Ap (roAp) stars the REE lines show the largest radial velocity pulsational amplitudes. In some stars only these line are pulsating while the lines of the Fe-peak elements exhibit either constant RV's or pulsate with negligible amplitudes. A joint abundance and pulsational analysis of $\gamma$ Equ (Ryabchikova et al. 2002) permitted us to derive REE abundance distributions with large concentrations of $\mathrm{Pr}$ and $\mathrm{Nd}$ in the upper atmosperic layers. NLTE Nd calculations presented by Mashonkina et al. (2005) 


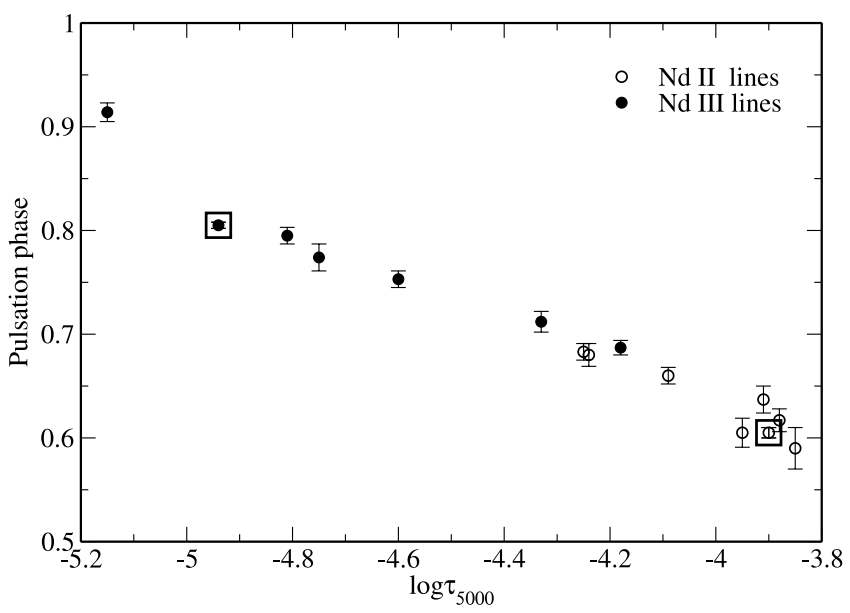

Figure 4. Optical depths of Nd II-Nd III line formation in $\gamma$ Equ with stratified Nd distribution presented by Mashonkina et al. (2005). Nd II $\lambda 6650$ and $\mathrm{Nd}$ III $\lambda 6550$ are marked by large open squares.

demonstrate the importance of NLTE effects for stratification studies of REEs in CP stars.

\section{Time-resolved spectroscopy}

Time-resolved spectroscopy is an advantage for the study of stellar pulsations, mainly due to higher amplitudes in the RV pulsations, and due to the selective character of the RV pulsations. As mentioned in the previous section, lines of different elements and even different ions of the same element show RV amplitudes which may differ by an order of magnitude. The study of pulsational line profile variations in lines formed at different parts of stellar atmosphere provide a way possibility to understand pulsation wave propagation through the whole atmosphere from the photosphere to the upper layers. Fig. 3 demonstrates significant differences in the pulsation picture observed from the lines of Nd II and Nd III and the similarity between Nd II and the H $\alpha$ core in $\gamma$ Equ. According to NLTE Nd calculations in $\gamma$ Equ (Mashonkina et al. 2005), Nd II and Nd III lines are formed in a $\mathrm{Nd}$ abundance layer. Therefore any changes in pulsation wave propagation takes place at smaller depths as is illustrated in Fig. 4.

The power of the modern spectrographs allows one to carry out time-resolved Zeeman spectroscopy to search for possible pulsational variations of the magnetic field. The first results for one of the brightest roAp stars, $\gamma \mathrm{Equ}$, are controversial. Leone \& Kurtz (2003) measured 12 min pulsational variations of the longitudinal magnetic field $B_{\ell}$ in four Nd III lines with the amplitude $\sim 110-240 \mathrm{G}$, while Kochukhov et al. (2004a) put an upper limit 40-60 $\mathrm{G}$ for possible $B_{\ell}$ amplitude based on the larger set of $\mathrm{Nd}$ III lines. The same limit was derived from non-pulsating lines of Fe-peak elements.

Careful measurements of the surface magnetic field $\left(B_{\mathrm{s}}\right)$ pulsational variations in the same star show no variations with the amplitude above 5-10 G (Kochukhov et al. 2004b). This conclusion was supported by Savanov et al. (2005).

\section{Lithium problem in cool CP stars}

An alternative identification of the famous spectral feature at $\lambda 6708$ as Ce II $\lambda 6708.099$ instead of to $\mathrm{Li} 1$ successfully resolved a problem of $\mathrm{Li}$ in atmospheres of post-AGB 

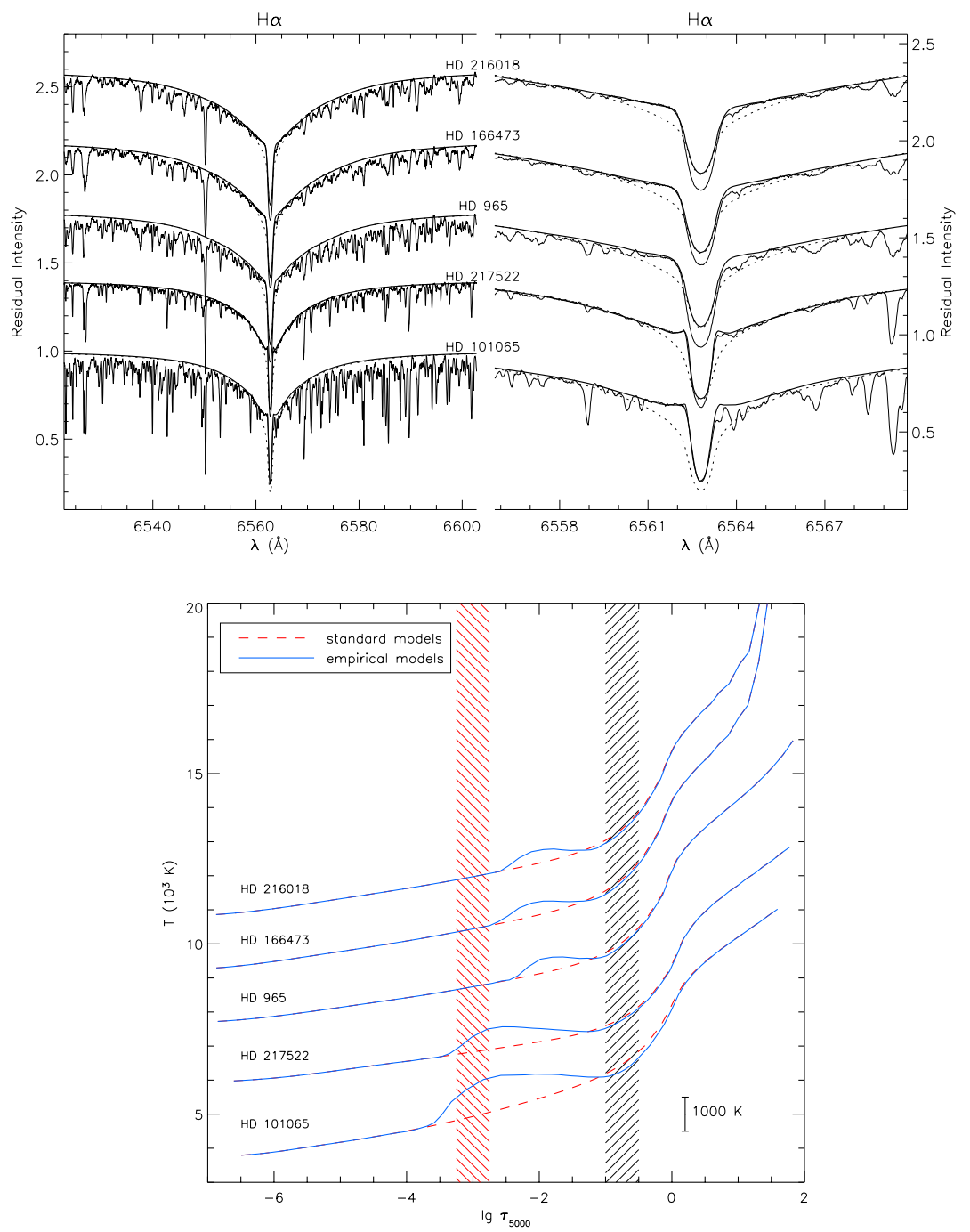

Figure 5. CWA effect in the cool roAp stars (top) and its explanation (bottom), from Kochukhov et al. (2003). The dashed line represents the standard model atmosphere while the full line shows the empirically derived temperature distribution. The left shadow region shows the approximate position of the REE jumps in roAp atmospheres obtained in NLTE approximation (see Mashonkina et al. 2005) while the right shadow region shows the position of Fe-peak abundance jumps derived empirically.

stars (Reyniers et al. 2002). It is tempting to replace Li by Ce in CP stars with their known large overabundances of REE (Nesvacil \& Hubrig 2005). Indeed, in some stars, for example, in HD $217522\left(T_{\text {eff }}=6750 \mathrm{~K}\right)$ the observed feature is entirely due to Ce II while in many other stars careful spectral synthesis shows that Lil remains the dominant contributer to the observed feature (Shavrina et al. 2005). It is also tempting to replace the Li 1 identification by any other unclassified line of REE because $\mathrm{Li}$ tends to be concentrated in spots at magnetic poles as REE do (Polosukhina et al. 2005, Kochukhov 2005). But in $\gamma$ Equ which shows one of the highest RV pulsational amplitudes derived from REE lines, a strong feature at $\lambda 6708$ does not show any significant pulsational variations, making doubtful the possible identification of the feature as a REE line. 


\section{Problems of the modelling of magnetic CP star atmosphere}

Until now analyses of CP stars were based on different versions of ATLAS LTE model atmosphere codes by Kurucz with homogeneous chemical compositions. In Section 4 I showed that cool CP star atmospheres are chemically inhomogeneous. Abundance gradients may alter the thermal structure of stellar atmosphere. Hydrogen line profiles may be used for diagnostics of plasma conditions. The study of high-resolution Balmer line profiles in spectra of a few cool CP stars revealed a departure of the observed profiles from those in normal stars and from the best theoretical ones in the transition region between line wing and line core. Instead of a smooth transition between the Stark wing and the Doppler core observed in normal stars an abrupt change in slope takes place in cool CP stars producing an unusually sharp line cores. The inner part of a Balmer line wing in a CP star is shallower than in the normal stars or in theoretical line profiles. This effect called core-wing anomaly (CWA) was first recognized by Cowley et al. (2001). Kochukhov et al. (2002) calculated empirical models where a temperature increases at intermediate atmospheric layers $-4 \leqslant \log \tau_{5000} \leqslant-1$ to obtain a good fit to the inner and outer wings as well as to the sharp cores of $\mathrm{H} \alpha$ and $\mathrm{H} \beta$ lines. Fig. 5 taken from Kochukhov et al. (2002) shows the results of their empirical modelling. I add to this plot an approximate range of the optical depths where empirically derived abundance jumps for Fe-peak and rare-earth elements take place. Observed bumps in the empirical temperature distributions fall between these jumps. Thus abundance gradients are responsible for the temperature bumps. The first self-consistent diffusion model atmosphere calculations (see LeBlanc \& Monin 2005) did not produce the needed temperature bumps, perhaps because of the lack of REEs in the diffusion calculations. The magnetic field which is not taken into account in these calculations may also alter the atmospheric structure of a CP star. The deviation of the observed $\mathrm{H} \alpha$ line profile from the theoretical one is observed for hotter CP stars with very large magnetic fields (see, e.g., HD 66318, Bagnulo et al. 2003, Fig.4). Here the inner part of the line wing is deeper in the observed profile contrary to that observed in cooler CP stars. Evidently, more elaborated atmosphere models are required to describe the most observed features in spectra of magnetic CP stars.

\section{Acknowledgements}

This work was supported by the FWF project $P$ 14984, by RFBR (grants 03-0216342, 04-02-16788) and by the Presidium RAS Programme "Nonstationary phenomena in astronomy".

\section{References}

Adelman, S.J., Malanushenko, V., Ryabchikova, T.A., Savanov, I. 2001, A\&AA 375, 982

Babel, J 1992, A\&A 258, 645

Bagnulo, S., Landstreet, J.D., Lo Curto, G., Szeifert, T., Wade, G.A. 2003, A $8 A$ 403, 449

Cowley, C.R., Hubrig, S., Ryabchikova T.A., Mathys, G., Piskunov, N., Mittermayer, P. 2001, A\& $A 367,939$

Bagnulo, S., Hensberge, H., Landstreet, J.D., Szeifert, T., Wade, G.A. 2004, A\&̊A 416, 1149

Gomez, A. E., Luri, X., Grenier, S., Figueras, F., North, P., Royer, F., Torra, J., Mennessier, M. O. 1998, A\&A 336, 953

Hubrig, S., North, P., Mathys, G. 2000a, ApJ 539, 352

Hubrig, S., North, P., Medici, A. 2000b, A\&A 359, 306

Kochuknov, O., Bagnulo, S., Barklem, P. 2002, ApJ 578, L75

Kochukhov, O., Ryabchikova, T. 2001, A\&A 374, 615

Kochukhov, O., Ryabchikova, T., Landstreet, J.D., Weiss, W.W 2004b, MNRAS 351, L34, 2004 
Kochukhov, O., Ryabchikova, T., Piskunov, N. 2004a, A\&A 415, L13

Kochukhov, O. 2005, These Proceedings, 433

Kurtz, D., Elkin, V.G., Mathys, G., Riley, J., Cunha, M.S., Shibahashi, H., Kambe, E. 2005, These Proceedings, 343

LeBlanc, F. 2003, in: L.A. Balona, H. Henrichs, \& T. Medupe (eds.), Int. Conf. on Magnetic Fields in $O, B$ and A Stars, ASP Conf. Ser, v.305, p.206

LeBlanc, D. \& Monin D., 2005, These Proceedings, 193

Leone, F., Kurtz, D.W. 2003, A\&3A 407, L67

Mashonkina, L., Ryabtsev, A., Ryabchikova, T. 2005, These Proceedings, 315

Michaud, G. 1970, ApJ 160, 641

Nesvacil, N., Hubrig, S. 2005, These Proceedings, FP21

Póhnl, H., Maitzen, H.M., Paunzen, E. 2003, A\& A 402, 247

Poloshukina, N., Shavrina, A, Drake, N., et al. 2005, These Proceedings, FP7

Pyper, D. M., Adelman, S. J. 2005, These Proceedings, 307

Pyper, D.M., Ryabchikova, T., Malanushenko, V. Kuschnig, R., Plachinda, S., Savanov, I. 1998, A $E$ A 339, 822

Reyniers, M., Van Winckel, H., Biémont, E., Quinet, P. A 8 A 395, L35

Ryabchikova, T., Piskunov, N., Kochukhov, O., Tsymbal, V., Mittermayer P., Weiss, W.W. 2002, A\& $A 384,545$

Ryabchikova, T., Wade, G.A., LeBlanc, F. 2003, in: N.E. Piskunov, W.W. Weiss, \& D.F. Gray (eds.), IAU Symp. 210 Modeling of Stellar Atmospheres, p. 301

Ryabchikova, T., Nesvacil, N., Weiss, W.W., Kochukhov, O., Stütz, Ch. 2004, A $\& A$ 423, 705

Ryabchikova, T., Leone, F., Kochukhov O., Bagnulo. S. 2005, These Proceedings, DP1

Savanov, I., Malanushenko, V.P., Ryabchikova, T.A. 1999, Astron. Lett. 25, 802

Savanov, I., Hubrig, S., Mathys, G., Ritter, A, Kurtz, D. W. 2005, This meeting, http://www.ta3.sk/IAUS224/PDF/GP10.pdf

Stẹpień, K. 2000, A\&A 353, 227

Wade, G.A., LeBlanc, F., Ryabchikova, T.A., Kudryavtsev, D. 2003, in: N.E. Piskunov, W.W. Weiss, \& D.F. Gray (eds.), IAU Symp. 210 Modeling of Stellar Atmospheres, CD-D7

\section{Discussion}

ALECIAN: In the stratified profiles you have shown, there is a step and then, a plateau. Is it possible to really check the extention of the plateau toward the deep layers because the line profiles are less sensitive to the deeper layers?

RyABCHIKOVA: An abundance plateau is assumed in our two-step abundance distribution model. However, the wings of strong lines with a large Stark effect are sensitive to element abundances in deeper layers below $\log \tau_{5000}=-1$ and even -0.5 . You can see it in our calculations for Si (Dimitrijević et al. 2003, A\&A 404, 1099, Fig.7).

Mathys: Note that in Gomeź et al. (1998) less than 20\% of stars have parallaxes known with relative accuracies better than $20 \%$. As to the way in which HD 66318 fits in the picture of deficiency of Ap stars close to the ZAMS, see my comment in yesterday afternoon's discussion.

BAgnulo: A comment to a question by Mathys: Probably the major problem with Hubrig et al. (2000) is that it is not possible to determine the evolutionary state of field stars with sufficient accuracy. At least, one can state weather the star is "young" or "old". By contrast, our work based on open cluster stars, although based on a poorer statistics, allows us to determine the age with much higher precision. In conclusion, I believe that the example of HD 66318 as well as some other ones not published yet, represents a clear counter-example to the Hubrig et al. hypothesis. 